\title{
Sterilisation - Einführung in die gesetzlichen Voraussetzungen
}

\author{
H. Schmid
}

\section{Sterilisationsgesetz}

Die Empfehlungen vom 12. Juni 2001 der Schweizerischen Akademie der Medizinischen Wissenschaften betreffend die Sterilisation von Menschen mit geistiger Behinderung (Ergänzung zu den Richtlinien von 1981) hielten fest: «In Einzelfällen kann es Gründe geben für die operative Sterilisation urteilsunfähiger Menschen mit geistiger Behinderung. In Anbetracht der heute noch bestehenden Gesetzeslücke soll derzeit in diesen Fällen eine Sterilisation unterbleiben. [...] Es ist Sache der aktiv gewordenen gesetzgeberischen Instanzen, Rahmenbedingungen zu formulieren, die den Eingriff als zulässig erklären.»

Das auf eine Parlamentarische Initiative von Felten zurückgehende Bundesgesetz vom 17. Dezember 2004 über Voraussetzungen und Verfahren bei Sterilisationen (Sterilisationsgesetz [im folgenden: SterG]; SR 211.111.1), in Kraft ab 1. Juli 2005, regelt nunmehr insbesondere die Voraussetzungen, unter denen eine Sterilisation zu Verhütungszwecken zulässig ist (Art. 1 SterG). Hauptanliegen des Erlasses ist die Regelung der Sterilisation dauernd Urteilsunfähiger (Art. 7 SterG); der Anwendungsbereich des Gesetzes ist aber umfassend.

\section{Legaldefinition der Sterilisation}

Das Sterilisationsgesetz definiert die Sterilisation in medizinischer und in normativer Hinsicht:

- Art. 2 Abs. 3 SterG enthält einen - selbstverständlichen - Arztvorbehalt: Die Sterilisation darf nur von einem Arzt vorgenommen werden. In Frage steht ein medizinischer Eingriff, mit dem die Fortpflanzungsfähigkeit einer Person auf Dauer aufgehoben wird (Art. 2 Abs. 1 SterG), indem die Ei- bzw. Samenleiter unterbrochen oder funktionsunfähig gemacht werden. «Auf Dauer» bedeutet nicht «endgültig»; dank mikrochirurgischer Operationstechnik besteht heute eine beachtliche Reversibilität der Sterilisation. Es ist - jedenfalls was dauernd Urteilsunfähige anbelangt - stets die Operationsmethode mit der grössten Refertilisierungsaussicht zu wählen (vgl. Art. 7 Abs. 2 Bst. f SterG).

- In normativer Hinsicht gelten Fälle nicht als Sterilisation, in denen die Aufhebung der Fortpflanzungsfähigkeit als Nebenfolge, d.h. als unvermeidliche Begleiterscheinung eines Heileingriffs, in Kauf genommen wird (Art. 2 Abs. 2 SterG). Der Arzt hat bloss die Pflicht, den Eingriff - sofern an einem Urteilsunfähigen vorgenommen - innerhalb von zehn Tagen der vormundschaftlichen Aufsichtsbehörde zu melden (Art. 10 Abs. 1 SterG).

\section{Sterilisation nach Personenkategorien}

\section{Volljährige}

\section{Urteilsfähige}

Die Sterilisation eines über 18jährigen Urteilsfähigen setzt voraus, dass er über den Eingriff umfassend informiert worden ist und diesem frei zugestimmt hat. Das zusätzliche Erfordernis einer schriftlichen Einwilligung (Art. 5 Abs. 1 SterG) will sicherstellen, dass er hinreichend aufgeklärt und in Kenntnis aller Umstände entscheidet.

Wer die Sterilisation durchführt, muss in der Krankengeschichte festhalten, aufgrund welcher Feststellungen er auf die Urteilsfähigkeit des Betroffenen in bezug auf den Eingriff geschlossen hat (Art. 5 Abs. 2 SterG). Es gilt zu verhindern, dass die Urteilsfähigkeit des zu Sterilisierenden leichtfertig angenommen wird. Der operierende Arzt kann sich auch auf Feststellungen des vorbehandelnden Arztes stützen.

\section{Urteilsfähige, aber Entmündigte}

Art. 6 SterG regelt die Voraussetzungen der Sterilisation über 18jähriger, urteilsfähiger und entmündigter Personen. 16- und 17jährige stehen zwar unter Vormundschaft wegen Unmündigkeit, sofern sie sich nicht unter elterlicher Sorge befinden (Art. 368 Abs. 1 ZGB); die Anordnung einer Vormundschaft über Unmündige ist jedoch keine Entmündigung im Sinne eines generellen Entzugs der Handlungsfähigkeit durch behördlichen Akt. Die Bevormundung wird, 
wenn sie wegen Unmündigkeit (und Fehlens elterlicher Sorge) eintritt, ohne vorausgehende Entmündigung angeordnet. Urteilsfähige 16und 17jährige unter Vormundschaft sind somit nach Art. 3 Satz 1 SterG geschützt, wonach die Sterilisation einer Person unter 18 Jahren verboten ist.

Art. 6 Abs. 1 Satz 1 SterG fordert die schriftlich erteilte, freie und umfassend aufgeklärte Einwilligung des zu Sterilisierenden - eine Voraussetzung, die bereits Art. 5 Abs. 1 SterG für die Sterilisation Urteilsfähiger festhält. Dementsprechend findet die Regelung nach Art. 6 Abs. 2 Bst. a SterG, wonach die Feststellungen in bezug auf die Urteilsfähigkeit in der Krankengeschichte festzuhalten sind, ihre Parallele in Art. 5 Abs. 2 SterG.

Volljährige, die - wiewohl urteilsfähig - unter Vormundschaft (Art. 369-372 ZGB) stehen, bedürfen eines besonderen Schutzes, weil sie wegen eines Schwächezustands im persönlichen Bereich ihre Interessen oft nicht selbst wahren können. Folglich hat der Arzt vor der Sterilisation zum einen die Zustimmung des gesetzlichen Vertreters (Art. 6 Abs. 1 Satz 2 SterG), zum andern die Zustimmung der vormundschaftlichen Aufsichtsbehörde einzuholen (Art. 6 Abs. 2 Bst. b SterG). Diese beiden Zustimmungen sollen den Entmündigten zusätzlich schützen.

In bezug auf die Urteilsfähigkeit des betroffenen Entmündigten ist stets - nicht nur bei Zweifeln - eine ärztliche Äusserung erforderlich, die von einem anderen als dem in Art. 6 Abs. 2 SterG genannten Operierenden stammt. Demnach muss die vormundschaftliche Aufsichtsbehörde eine ärztliche Zweitmeinung einholen (Art. 6 Abs. 3 Satz 1 SterG). Erscheint ihr dies nicht genügend, so darf sie ein psychiatrisches Gutachten anordnen (Art. 6 Abs. 3 Satz 2 SterG). Für den Betroffenen ist eine psychiatrische Begutachtung belastend; die Massnahme ist somit nur gerechtfertigt, wenn trotz ergänzender Abklärungen erhebliche Zweifel an der Urteilsfähigkeit fortbestehen. In den meisten Fällen wird die Meinung eines anderen Arztes ausreichen, um Bedenken der vormundschaftlichen Aufsichtsbehörde zu entkräften. Jedenfalls darf ein psychiatrisches Gutachten nicht systematisch angeordnet werden, nur weil der Betroffene unter Vormundschaft steht.

\section{Vorübergehend Urteilsunfähige}

Bei voraussichtlich nur vorübergehend Urteilsunfähigen ist die Sterilisation nicht zu rechtfertigen (Art. 4 SterG), gilt es doch zu verhindern, dass bei solchen Betroffenen während dieses zeitweiligen Zustands die Fortpflanzungsfähigkeit auf Dauer aufgehoben wird.
Wenn gemäss Prognose die Wiedererlangung der Einwilligungsfähigkeit zwar nicht völlig ausgeschlossen ist, aber nach ärztlicher Voraussicht die Urteilsunfähigkeit mit an Sicherheit grenzender Wahrscheinlichkeit bestehen bleibt, dann gelten die Regeln über dauernd Urteilsunfähige (siehe unten).

\section{Minderjährige}

Der Gesetzgeber geht davon aus, dass jungen Menschen vielfach die Reife fehlt, um den Sterilisationseingriff in seiner vollen Tragweite $z u$ erfassen und in bezug auf die Elternschaft eine Lebensplanung vorzunehmen. Die Sterilisation einer Person unter 18 Jahren ist deshalb grundsätzlich verboten (Art. 3 Satz 1 SterG) - die Grenze ist in zeitlicher Hinsicht mit Beginn des 18. Geburtstags (00 Uhr früh) erreicht. Eine Ausnahme gilt bloss in bezug auf über 16jährige, dauernd Urteilsunfähige: Sie können unter einschränkenden Voraussetzungen sterilisiert werden (Art. 3 Satz 2 i.Verb.m. Art. 7 SterG).

\section{Dauernd Urteilsunfähige}

\section{Formale Voraussetzung}

Der Arzt darf den Eingriff nur vornehmen, wenn die vormundschaftliche Aufsichtsbehörde zugestimmt hat (Art. 7 Abs. 2 Bst. g in Verbindung mit Art. 8 Abs. 1 SterG). Die Kantone haben von Bundesrechts wegen die Vormundschaftsbehörde und in bezug auf die Instanzenzahl eine, höchstens zwei Aufsichtsbehörden zu bestimmen (Art. 361 ZGB). Kantone, die zwei vormundschaftliche Aufsichtsbehörden vorsehen, werden in der Regel festhalten, dass in erster Instanz die untere vormundschaftliche Aufsichtsbehörde über die Sterilisation entscheidet. Die territoriale Kompetenzausscheidung ist Sache der Kantone.

\section{Materielle Voraussetzungen}

Die vormundschaftliche Aufsichtsbehörde wird bei ihrem Entscheid prüfen, ob folgende Voraussetzungen kumulativ (vgl. das Wort «und» in Art. 7 Abs. 2 Bst. f SterG) vorliegen:

- Der Vorrang anderer Verhütungsmittel besagt, dass die Zeugung und die Geburt eines Kindes nicht durch geeignete andere Verhütungsmethoden oder - wohl eher lebensfremd - durch freiwillige Sterilisation des urteilsfähigen Partners verhindert werden können (Art. 7 Abs. 2 Bst. b SterG). In erster Linie sollen im Sinn des Verhältnismässigkeitsprinzips reversible kontrazeptive Verfahren Anwendung finden.

- $\quad$ Die betroffene Person ist über 16jährig (Art. 7 Abs. 1 SterG). 
- Nach ärztlicher Einschätzung ist sie - ohne Aussicht, jemals die Urteilsfähigkeit zu erlangen (Art. 7 Abs. 2 Bst. e SterG) - dauernd urteilsunfähig (Art. 7 Abs. 1, Art. 8 Abs. 2 Bst. c SterG); zudem würde eine Schwangerschaft ihre Gesundheit erheblich gefährden (Art. 7 Abs. 2 Bst. d SterG).

- Der Entwurf zum Sterilisationsgesetz erlaubte den Eingriff bloss «im ausschliesslichen Interesse der betroffenen Person» (Art. 7 Abs. 2 Bst. a E SterG). Demgegenüber setzte sich im Parlament eine offenere Formulierung durch, wonach zwar im Interesse der betroffenen Person, aber «nach den gesamten Umständen» entschieden werden muss (Art. 7 Abs. 2 Bst. a SterG). Ausschlaggebend war die Erkenntnis, dass auch die Eltern und Geschwister der geistigbehinderten Person von der Problematik betroffen sind, denn mit einer Schwangerschaft kommen erhebliche Zusatzbelastungen auf diese Personen zu. Und letztlich sind auch die Interessen eines allfälligen Kindes der geistigbehinderten Person zu bedenken. Deshalb geht es für den Gesetzgeber zu weit, die Interessen Dritter explizit als unerheblich zu bezeichnen.

Nach dem Entwurf zum Sterilisationsgesetz war die Sterilisation zudem unzulässig, wenn die betroffene Person «Ablehnung gegen den Eingriff geäussert hat» (Art. 7 Abs. 2 Bst. a E SterG). Auch eine unbestimmte Angst vor dem ärztlichen Eingriff galt als rechtswirksamer Widerspruch. Der Arzt hätte die betroffene Person nicht einmal auf medikamentöse Weise, sondern bloss in einem klärenden Gespräch dabei unterstützen dürfen, Befürchtungen in bezug auf den vorzunehmenden Eingriff als solchen zu überwinden. Die Selbstbestimmung wäre bis zur letzten Minute beachtlich gewesen. Ein solches Konzept eines «natürlichen (entgegenstehenden) Willens» einer urteilsunfähigen Person hätte aber faktisch zu einem Sterilisationsverbot geführt, weil viele Menschen mit einer schweren geistigen Behinderung unverhält- nismässige Angst vor medizinischen Massnahmen und Untersuchungen haben. Die Folgen einer unterlassenen Sterilisation Schwangerschaft, Geburt oder Abtreibung einerseits, Beschränkung der persönlichen Freiheit zwecks Verhinderung von sexuellen Kontakten andererseits - wären für die betroffene Person aber noch belastender gewesen. Das Parlament lehnte die erwähnte Formulierung des Entwurfs denn auch ab.

- Sterilisationsvoraussetzung nach dem Entwurf war eine ernsthafte Gefährdung der körperlichen oder seelischen Gesundheit der betroffenen Person, und zwar infolge der Schwangerschaft, der Elternschaft oder der unvermeidlichen Trennung vom Kind (Art. 7 Abs. 2 Bst. d E SterG). Das Parlament gelangte aber zur Überzeugung, ein Sterilisationsverbot ausgerechnet im Fall emotionaler Indifferenz infolge geistiger Behinderung mache keinen Sinn. Die Voraussetzung wurde deshalb objektiver formuliert und die sorgerechtliche Indikation der unvermeidlichen Trennung vom Kind selbständig ausgestaltet, und zwar entsprechend der Formulierung: Wenn «nach der Geburt die Trennung vom Kind unvermeidlich wäre, weil die Elternverantwortung nicht wahrgenommen werden kann» (Art. 7 Abs. 2 Bst. d SterG).

- Die Sterilisation ist nur zulässig, wenn «mit der Zeugung und der Geburt eines Kindes zu rechnen ist» (Art. 7 Abs. 2 Bst. c SterG). Die vorsorgliche Sterilisation einer sexuell inaktiven Frau im Hinblick auf die abstrakte Gefahr einer Vergewaltigung ist somit unzulässig, was freilich nicht bedeutet, dass eine dauernd urteilsunfähige Person erst ungeschützten sexuellen Kontakt pflegen muss, um die Sterilisationsvoraussetzungen zu erfüllen. Da heute Heime, Wohngruppen usw. für Geistigbehinderte kaum mehr nach Geschlechtern getrennt geführt werden, ist ein rechtzeitiger Schutz vor ungewollter Schwangerschaft unabdingbar, können Betreuer doch kaum voraussehen, wann genau Beziehungen intimen Charakter annehmen. 